\title{
Modelling of flame temperature of solution combustion synthesis of nanocrystalline calcium hydroxyapatite material and its parametric optimization
}

\author{
SAMIR K GHOSH ${ }^{\dagger}$, SUKHOMAY PAL*, SUJIT K ROY ${ }^{\dagger \dagger}$, SURJYA K PAL and \\ DEBABRATA BASU ${ }^{\dagger \dagger}$ \\ Department of Mechanical Engineering, Indian Institute of Technology, Kharagpur 721 302, India \\ ${ }^{\dagger}$ Biomedical Engineering Department, Netaji Subhas Engineering College, Kolkata 700 032, India \\ ${ }^{\dagger \dagger}$ Central Glass and Ceramic Research Institute, Kolkata 700 032, India
}

MS received 16 February 2009

\begin{abstract}
Hydroxyapatite (HAp), an important bio-ceramic was successfully synthesized by combustion in the aqueous system containing calcium nitrate-di-ammonium hydrogen orthophosphate-urea. The combustion flame temperature of solution combustion reaction depends on various process parameters, and it plays a significant role in the phase formation, phase stability and physical characteristics of calcium hydroxyapatite powder. In this work, an attempt has been made to evaluate the influence of each selected process parameters on the flame temperature as well as physical characteristics of powder, and to select an optimal parameters setting using Taguchi method. A regression model has also been developed to correlate the input parameters, viz. batch size, diluents, fuel to oxidizer ratio and initial furnace temperature, with flame temperature of the solution combustion reaction. The adequacy of the developed model has been checked using analysis of variance technique.
\end{abstract}

Keywords. Solution combustion; combustion flame temperature; design of experiment; Taguchi's technique; response surface methodology; regression analysis.

\section{Introduction}

Calcium phosphate-based bioceramics have been in use in medicine and dentistry for last 40 years (Hench 1991). With the growth in medical science and advancements in surgical techniques, there is an increasing demand for artificial bone implants. This happens due, on one hand, to a limited supply of auto-graft material and, on the other, the health risks associated with the use of allograft (Hing et al 1999). The most used calcium phosphate materials in medical field is hydroxyapatite (HAp), $\mathrm{Ca}_{10}\left(\mathrm{PO}_{4}\right)_{6}(\mathrm{OH})_{2}$, since it is the most similar material to the mineral component of biological bones. It shows excellent biocompatibility with hard tissues, and also with skin and muscle tissues (Nandi et al 2008a). Formation of chemical bond with the host tissue offers HAp a greater advantage in clinical applications over most other bone substitutes, such as allografts or metallic implants (Raemdonck et al 1984). Unfortunately, its low fracture toughness $\left(0.8-1.2 \mathrm{MPa} \mathrm{m}^{1 / 2}\right)$ and flexural strength $(<130 \mathrm{MPa})$ (Willman 1996) currently restrict its use to small unloaded implants, powders, coatings, and lowloaded porous implants (Kanazawa 1989; Hench 1998).

\footnotetext{
*Author for correspondence (spalsa08@yahoo.co.in)
}

According to its chemical formula, stoichiometric HAp should have the $\mathrm{Ca} / \mathrm{P}$ atom ratio of 1.67 . The stability limit of the apatitic structure in HAp has been the subject of a number of studies, covering a rather wide range of compositions (Wang and Chaki 1993). The apatitic crystal structure can be preserved with $\mathrm{Ca} / \mathrm{P}$ ratios as low as 1.5 , and are called calcium-deficient or non-stoichiometric. These materials exhibit thermal instabilities at characteristic temperatures (Fanovich et al 2001). So the processing temperature is carefully controlled to retain the phase stability of HAp crystal.

Various routes to synthesize HAp powders have been developed. Frequently used technique is the wet chemical precipitation (Sung et al 2004). Others include hydrothermal reaction (Liu et al 1997), mechanochemicalhydrothermal synthesis (Suchanek et al 2002) and sol-gel synthesis (Liu et al 2001, 2002). However, these methods have several disadvantages including difficulty to maintain the $\mathrm{pH}$ value above 9 during the initial solution, formation of calcium deficient HAp, which after further heat treatment easily decomposes to $\beta$-TCP.

Recently, solution combustion method is widely used to synthesize various nanocrystalline oxide ceramic powders (Kingsley and Patil 1988; Manoharan and Patil 1992; Balmer et al 1995; Fumo et al 1996). The key 
features of this method are its ability to produce exact stoichiometric phases with high purity, better homogeneity and ultra fine powders in a single step. Tas (2000) successfully synthesized calcium phosphate powders by using novel synthetic body fluid solutions via the selfpropagating combustion synthesis method. Varma et al (1998) also synthesized calcium phosphate powders by a polymeric combustion method via a solution of calcium nitrate and ethyl phosphate. Synthesis of HAp and $\beta$-TCP powders and their composites through aqueous solution combustion technique are also done (Ghosh et al 2004, 2008; Nandi et al 2008b).

The basis of combustion synthesis technique comes from the thermo-chemical concepts used in the field of propellants and explosives chemistry. In this field, calculation of effective constituents of a fuel to oxidizer mixture is of paramount importance. The total oxidizing and reducing valences of the oxidizer and fuel were determined as proposed by Jain et al (1981). According to him the maximum heat is released when the oxidizer to fuel ratio is stoichiometric $(\mu=1)$. Stoichiometric composition of oxidizer to fuel is calculated using the total oxidizing and reducing valences of the reactants, which serve as numerical coefficient for stoichiometric balance so that equivalent ratio is unity $(\mu=1)$ and energy released in the combustion reaction is maximum.

In the solution combustion process molecular level mixing of the reactants occurred and the combustion reaction was so fast that it was assumed to be adiabatic. The maximum temperature generated theoretically in a combustion reaction is called adiabatic flame temperature $\left(T_{\mathrm{f}}\right)$. But in practice, the combustion reaction of HAp synthesis was not perfectly adiabatic because external oxygen supply was essential to phase formation of the HAp powder (Ghosh et al 2004). For this reason, the measured flame temperature of the combustion reaction was observed to be significantly lower than that of the actual adiabatic flame temperature. From previous work (Ghosh et al 2004), it was found that some process parameters, viz. batch size $(B)$, diluents $(D)$, fuel to oxidizer ratio $(\mu)$ and initial furnace temperature $(F)$ significantly controlled the measured combustion flame temperature $\left(T_{\mathrm{c}}\right)$ (Hong et al 1994; Venkatachari et al 1995; Schafer et al 1997; Mukasyan et al 2001; Purohit et al 2001a, b; Ghosh et al 2004). This measured combustion flame temperature has a significant effect on the phase formation and phase stability as well as product characteristics (Segadges et al 1998; Feng et al 2005). It was also found that a better result was obtained when the measured combustion flame temperature was near about $780^{\circ} \mathrm{C}$ (Ghosh et al 2004). For this reason this temperature was chosen as target flame temperature in this work. Higher combustion flame temperature may lead to sintering effect of the powder particles. Several researchers have investigated the effects of $\mu$ (Schafer et al 1997; Mukasyan et al 2001; Purohit et al 2001a, b) and initial furnace temperature and precursor batch size (Hong et al 1994; Venkatachari et al 1995) on the combustion flame temperature as well as product characteristics of the solution combustion process. Most of the reports have shown that combustion flame temperature increases with increasing initial furnace temperature and batch size which leads to the sintering effects of the particles resulting in reduction of the powder surface area. Investigations of $\mu$ on the combustion flame temperature have shown that both fuel lean $(1<\mu)$ and fuel rich $(\mu>1)$ systems produce combustion flame with lower temperature than that of stoichiometric fuel $(\mu=1)$. Investigation reports (Schafer et al 1997; Mukasyan et al 2001) suggested fuel rich combustion system to synthesize nanocrystalline ceramic powders due to low flame temperature and large volume gas generation. But no work has been reported on the effects and relations of all process parameters on the measured combustion flame temperature as well as product characteristics at a time.

In this work, two types of design of experimental (DOE) technique, such as Taguchi and response surface method (RSM) have been applied to perform the experiments. Taguchi $L_{16}$ orthogonal array was used to evaluate the influence of different process parameters, viz. batch size, diluents, fuel to oxidizer ratio and initial furnace temperature, on the measured combustion flame temperature, and optimization of the selected process parameters in terms of target flame temperature $\left(T_{\mathrm{c}}=780^{\circ} \mathrm{C}\right)$ has also been done. Analysis of variance (ANOVA) technique was applied to investigate the influence of different solution combustion process parameters on the $T_{\mathrm{c}}$ as well as powder characteristics. Response surface methodology was used to explore the relationships between several explanatory variables and response variable. Three different types of regression models, viz. first degree liner model, second degree surface model, and power model, were developed for prediction of the flame temperature by using different process parameters. The adequacy of the developed models has been checked using ANOVA technique.

\section{Experimental}

The choice of an experimental design depends on the objectives of the experiment and the number of factors to be investigated. The objective of the present work was (i) to optimize the process parameters and to study the parametric influence on response, and (ii) to determine and represent the cause and effect relationship between true mean response and input control variables. To fulfill the above mentioned criteria, we used Taguchi's technique and response surface methodology, respectively. Taguchi's technique was mainly used for solving optimization problem and to study the parametric influence on responses (Roy 2001). RSM is a technique to represent the cause 
and effect relationship between true mean response and input control variables influencing the response as a two or three dimensional hyper surface (Myers and Montgomery 1995; Pal et al 2007). Taguchi's and RSM technique have been discussed in $\$ \S 2.1$ and 2.2 , respectively.

\subsection{Taguchi's technique for parametric optimization}

Dr Genichi Taguchi is a Japanese scientist who developed a technique based on orthogonal array of experiments, which provide much reduced variance for the experiment with optimum setting of process control parameters. This technique has been widely used in different fields of engineering to optimize the process parameters, and to determine the impacts of different parameters within the combination of design parameters. Thus the integration of design of experiment with parametric optimization of process is achieved in the Taguchi method (This will provide desired results). In this case the desired result refers to the target flame temperature of solution combustion reaction.

An orthogonal array provides a set of well balanced experiments and Taguchi's signal-to-noise $(S / N)$ ratios, which are logarithmic functions of the desired output, serve as objective functions for optimization. It helps to learn the whole parameters space with a small number (minimum experimental runs) of experiments only. These are used to study the effects of control factors and noise

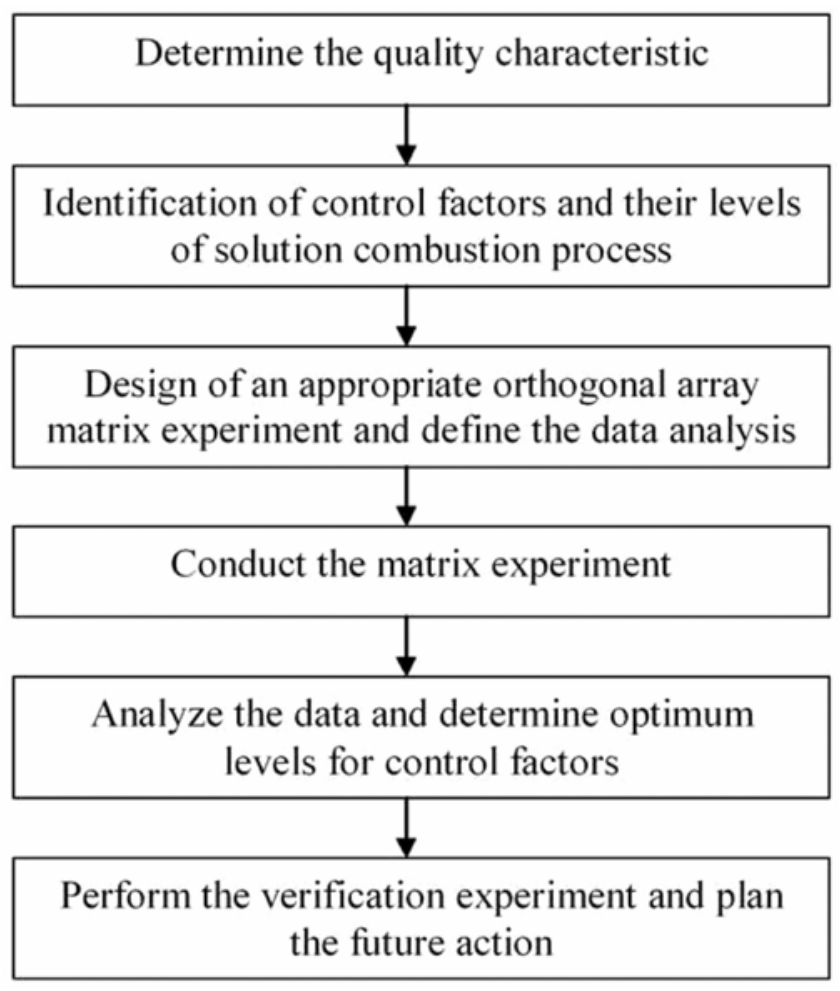

Figure 1. Flow chart showing the steps of Taguchi approach. factors, and to determine the best quality characteristics for particular applications (Roy 2001). In order to apply the Taguchi method for the present investigation, the following five phases have been planned, which is shown in figure 1 .

2.1a Identification of control factors and their levels: A controlled factor is a characteristic that can be controlled in the product or process subjected to designing. From the available literature (Hong et al 1994; Venkatachari et al 1995; Schafer et al 1997; Mukasyan et al 2001; Purohit et al 2001a, b) and previous works (Ghosh et al 2004, 2008; Nandi et al 2009), the predominant factors that are having greater influence on the measured combustion flame temperature $\left(T_{\mathrm{c}}\right)$ are used in this work. Such factors are fuel to oxidizer ratio, initial furnace temperature, precursor batch size or quantity and percentage of preformed HAp powder addition or diluents. Adequate numbers of trial experiments have been carried out in the laboratory. For each trial run, combustion flame temperature has been measured and inspected to identify the working limits of the control factors or process parameters. From the above investigations, the following observations have been made:

(i) It has been observed that below and above a certain limit of fuel to oxidizer ratio $(\mu)$ incomplete combustion reactions occurred. The lower value of $\mu$ was identified as 0.8 and for the upper value of $\mu$ as 3.2 .

(ii) The initial furnace temperature was found to be a very important reaction parameter. The lower value of furnace temperature was selected as $400^{\circ} \mathrm{C}$ and the upper value as $700^{\circ} \mathrm{C}$.

(iii) The precursor batch size was also identified as an important parameter in this method. If the reaction container having the capacity, $150 \mathrm{ml}$, contain below $10 \mathrm{ml}$ (3.00 $\mathrm{g}$ of HAp) of experimental solution, then it does not produce the spontaneous combustion reaction and above $15 \mathrm{~g}$ of HAp the reaction tends to be explosive in nature. So, for controlled and stable combustion reaction lower and upper values selected in this experiment are $3.26 \mathrm{~g}$ and $13 \cdot 17 \mathrm{~g}$, respectively.

(vi) Alteration of combustion temperature was also observed with addition of different quantity of fine preformed HAp powder in the precursor batch. The HAp powder acts as a diluent and it takes the reaction heat from the system, resulting in the reduction in combustion temperature. The lower and upper limits of diluent selected in this experiment are 0 and $30 \%$ of theoretical yield of HAp powder, respectively; because above $30 \%$ of the value leads to unstable combustion flame. The control factors or process parameters and their different levels with their notations are shown in table 1 .

2.1b Design of an appropriate orthogonal array matrix: Taguchi's orthogonal arrays are experimental designs that usually require only a fraction of the full factorial combi- 
Table 1. Process parameters and their different levels for calcium nitrate-DAP-urea system.

\begin{tabular}{|c|c|c|c|c|c|c|c|c|c|}
\hline \multirow[b]{2}{*}{ S1. no. } & \multirow[b]{2}{*}{ Parameter } & \multirow[b]{2}{*}{ Notation } & \multicolumn{4}{|c|}{ Taguchi } & \multicolumn{3}{|c|}{ RSM (levels) } \\
\hline & & & Level 1 & Level 2 & Level 3 & Level 4 & -1 & 0 & 1 \\
\hline 1 & Batch size (g) & $B$ & $3 \cdot 26$ & $6 \cdot 82$ & $9 \cdot 74$ & $13 \cdot 17$ & $4 \cdot 9$ & $7 \cdot 32$ & $9 \cdot 74$ \\
\hline 2 & Diluents (\%) & $D$ & 0 & 10 & 20 & 30 & 10 & 20 & 30 \\
\hline 3 & Fuel to oxidizer ratio & $\mu$ & $0 \cdot 80$ & $1 \cdot 00$ & 1.90 & $3 \cdot 20$ & $0 \cdot 60$ & $0 \cdot 90$ & $1 \cdot 20$ \\
\hline 4 & Initial furnace temperature $\left({ }^{\circ} \mathrm{C}\right)$ & $F$ & 400 & 500 & 600 & 700 & 400 & 500 & 600 \\
\hline
\end{tabular}

Table 2. Design matrix $L_{16}$ with corresponding responses $\left(T_{\mathrm{c}}\right)$ and $S / N$ ratio.

\begin{tabular}{lllllcc}
\hline Experimental run & $B$ & $D$ & $\mu$ & $F$ & $\begin{array}{c}\text { Measured combustion } \\
\text { flame temperature, } T_{\mathrm{c}}\left({ }^{\circ} \mathrm{C}\right)\end{array}$ & $S / N$ ratio $(\mathrm{d} B)$ \\
\hline 1 & 1 & 1 & 1 & 1 & 678 & $20 \cdot 0928$ \\
2 & 1 & 2 & 2 & 2 & 702 & $22 \cdot 5648$ \\
3 & 1 & 3 & 3 & 3 & 681 & $20 \cdot 3700$ \\
4 & 1 & 4 & 4 & 4 & 647 & $17 \cdot 6011$ \\
5 & 2 & 1 & 2 & 3 & 793 & $38 \cdot 6454$ \\
6 & 2 & 2 & 1 & 4 & 777 & $51 \cdot 2930$ \\
7 & 2 & 3 & 4 & 1 & 722 & $25 \cdot 2545$ \\
8 & 2 & 4 & 3 & 2 & 727 & $33 \cdot 7448$ \\
9 & 3 & 1 & 3 & 4 & 703 & $51 \cdot 3265$ \\
10 & 3 & 2 & 4 & 3 & 766 & $37 \cdot 8513$ \\
11 & 3 & 3 & 1 & 2 & 755 & $32 \cdot 7531$ \\
12 & 3 & 4 & 2 & 1 & 807 & $32 \cdot 3740$ \\
13 & 4 & 1 & 4 & 2 & 797 & $36 \cdot 3374$ \\
14 & 4 & 2 & 3 & 1 & 852 & $24 \cdot 0975$ \\
15 & 3 & 2 & 4 & 801 & $34 \cdot 5240$ \\
16 & 4 & 4 & 1 & 3 & & \\
\hline
\end{tabular}

nations. The columns of the arrays are balanced and orthogonal. This means that in each pair of columns, all factor combinations occur at the same number of times. Orthogonal designs allow estimating the effect of each factor on the response independently of all other factors. In this work, an $L_{16}$ orthogonal array was used which composed of four process parameters with four levels of each. The design matrix, in coded form, is shown in table 2 .

2.1c Experiment conducted: The experiments were conducted based on Taguchi's orthogonal array design, which has been mentioned in the previous section. The analytical grade raw materials used in these experiments were calcium nitrate tetrahydrate $\left(\mathrm{Ca}\left(\mathrm{NO}_{3}\right)_{2} \cdot 4 \mathrm{H}_{2} \mathrm{O}\right)$ (S.D. Fine-Chem. Ltd., India) and di-ammonium hydrogen ortho-phosphate (DAP), $\left[\left(\mathrm{NH}_{4}\right)_{2} \mathrm{HPO}_{4}\right]$ (S.D. Fine-Chem. Ltd., India) for preparation of calcium hydroxyapatite (HAp) powders. Analytical grade urea $\left[\mathrm{CO}\left(\mathrm{NH}_{2}\right)_{2}\right]$ (Glaxo, Qualigens, India) was used as the fuel. For synthesizing HAp, aqueous stock solutions of calcium nitrate tetrahydrate $(2.72 \mathrm{M})$ and DAP $(2.09 \mathrm{M})$ were first mixed slowly and a value of $1.67 \mathrm{Ca} / \mathrm{P}$ atomic ratio was maintained strictly with continuous stirring, subsequently concentrated nitric acid was added dropwise to dissolve the resulting white precipitate. A predetermined amount of solid fuel was added to the clear solution and homogenized by stirring with a magnetic stirrer for $30 \mathrm{~min}$ at room temperature. Phase pure synthesized submicron grade preformed HAp powder was used as diluent. Addition of percentage of diluent was calculated on the basis of theoretical yield product of HAp powder and was mixed with the precursor solution.

One glass of ceramic-coated half sphere mild steel (dia. $\sim 80 \mathrm{~mm}$, volume 130 c.c.) container containing the solution was introduced into a muffle furnace preheated to the desired temperature $\left(400-700^{\circ} \mathrm{C}\right)$. Flame temperatures of different experimental batches were recorded by using a $Z$-trend paperless chart recorder (Honeywell, USA) with the help of chromel-alumel ( $k$-type) thermocouple. The output flame temperatures obtained from various parametric conditions is shown in table 2 . The heat evolved during the reaction sustained itself and proceed to completion without requiring any further heat from an external source.

\subsection{Application of response surface methodology for developing response models}

Response surface methodology is a collection of mathematical and statistical techniques in design or data analysis 
that enhance the exploration of a region of design variables in one or more responses. The experimental strategy and analysis in RSM revolves around the assumption that a response is a function of a set of design variables and that the function can be approximated in some region of the design variables by a polynomial model. Detailed description of RSM is out of the scope of this article; interested readers may refer to the relevant technical book (Myers and Montgomery 1995).

In this investigation, RSM was also used to understand the effect of different solution combustion synthesis of calcium hydroxyapatite process parameters on the flame temperature and to develop a model for the flame temperature prediction. A three-level, four-factor, central composite, rotatable design matrix with seven centre points was used to optimize the required number of experiments. This design requires thirtyone experimental runs. A commercially available software package, MINITAB ${ }^{\mathrm{TM}}$, USA, 2000, was used to setup the design matrix. Table 1 presents the range of factors considered and table 3 shows 31 sets of coded conditions of the design matrix.

\subsection{Powder characterization}

The crystallinity and phase identification of the powders were carried out by using Philips X-ray diffractometer (XRD) (Philips Analytical, X'Pert, 1830, The Netherlands) with $\mathrm{Cu}-\mathrm{K}_{\alpha \mathrm{l}}$ radiation and $\mathrm{Ni}$-filter. The average crystallite size of the powders was determined by the line-broadening method by using the Scherrer formula, as given below:

$$
D=\frac{0 \cdot 9 \lambda}{\beta \cos \theta},
$$

where $D$ is the average crystallite size in $\mathrm{nm}, \lambda$ $(\sim 0.154056 \AA \mathrm{nm})$ the wavelength of the X-ray radiation, $\theta$ the Bragg's angle and $\beta$ the full width at half maximum (FWHM) of the strongest diffraction peak of HAp (211) observed.

Scanning electron microscope (SEM) (Leo 430i, UK) and field emission scanning electron microscope (FESEM) (Supra-35 VP, Carl Zeiss, Germany) of a few selected samples were used to investigate the particles size, shape and morphology of as synthesized powders.

\section{Results and discussion}

\subsection{Analysis of data and determination of optimum} levels using $S / N$ ratio

In order to evaluate the influence of each selected factor on the response and to evaluate optimal parameters setting, the Taguchi method uses a statistical measure of performance, called signal-to-noise $(S / N)$ ratio. The $S / N$ ratio is a performance measure to select control levels that best cope with noise. The signals have indicated that the effect on the average responses and the noises were measured by the influence on the deviations from the average responses, which would indicate the sensitiveness of the experiment output to the noise factors. The ratio depends on the quality characteristics of the pro$\mathrm{duct} /$ process to be optimized (Roy 2001). The standard $S / N$ ratios generally used are nominal-is-best (NB), lower-thebetter (LB) and higher-the-better (HB). The appropriate $S / N$ ratio must be chosen using previous knowledge and understanding of the process. In this research, the characteristic value of the process is chosen as combustion flame temperature. Since a good result is obtained when response is nearer to the target because proper phase formation of HAp takes place when the flame temperature is between 750 and $800^{\circ} \mathrm{C}$ (Schafer et al 1997; Zahid 1998). In the present research, we used $780^{\circ} \mathrm{C}$ flame temperature as the target $T_{\mathrm{c}}$. Hence, NB criterion has been chosen. The values of $S / N$ ratio for NB criteria were calculated as follows:

$$
S / N=10 \log _{10}\left(\frac{y_{\mathrm{avg}(i)}^{2}}{\sigma_{i}^{2}}\right),
$$

where, $y_{\mathrm{avg}(i)}$ is the average of $i$ th experimental output and target value and $\sigma_{i}$ the standard deviation of $i$ th response.

\begin{tabular}{|c|c|c|c|c|c|}
\hline Exp. no. & $B$ & $D$ & $\mu$ & $F$ & $T_{\mathrm{c}}$ \\
\hline 1 & -1 & -1 & -1 & -1 & 727 \\
\hline 2 & 0 & -1 & 0 & 0 & 747 \\
\hline 3 & -1 & 1 & -1 & 1 & 697 \\
\hline 4 & 1 & 0 & 0 & 0 & 817 \\
\hline 5 & 0 & 0 & 0 & -1 & 724 \\
\hline 6 & 1 & -1 & -1 & 1 & 803 \\
\hline 7 & 0 & 0 & -1 & 0 & 723 \\
\hline 8 & -1 & 1 & 1 & 1 & 723 \\
\hline 9 & -1 & -1 & 1 & 1 & 756 \\
\hline 10 & 0 & 0 & 0 & 0 & 734 \\
\hline 11 & 1 & 1 & -1 & 1 & 767 \\
\hline 12 & 0 & 0 & 0 & 0 & 729 \\
\hline 13 & 0 & 0 & 0 & 0 & 737 \\
\hline 14 & 0 & 0 & 0 & 0 & 739 \\
\hline 15 & -1 & 0 & 0 & 0 & 719 \\
\hline 16 & 1 & -1 & 1 & -1 & 833 \\
\hline 17 & -1 & -1 & 1 & -1 & 737 \\
\hline 18 & 0 & 1 & 0 & 0 & 714 \\
\hline 19 & 0 & 0 & 0 & 0 & 731 \\
\hline 20 & 1 & -1 & -1 & -1 & 781 \\
\hline 21 & 1 & 1 & 1 & -1 & 787 \\
\hline 22 & 0 & 0 & 1 & 0 & 727 \\
\hline 23 & 0 & 0 & 0 & 1 & 744 \\
\hline 24 & 1 & 1 & -1 & -1 & 743 \\
\hline 25 & 0 & 0 & 0 & 0 & 733 \\
\hline 26 & 1 & -1 & 1 & 1 & 857 \\
\hline 27 & 0 & 0 & 0 & 0 & 739 \\
\hline 28 & -1 & 1 & 1 & -1 & 712 \\
\hline 29 & -1 & 1 & -1 & -1 & 683 \\
\hline 30 & 1 & 1 & 1 & 1 & 802 \\
\hline 31 & -1 & -1 & -1 & 1 & 729 \\
\hline
\end{tabular}

Table 3. Design matrix and experimental results. 


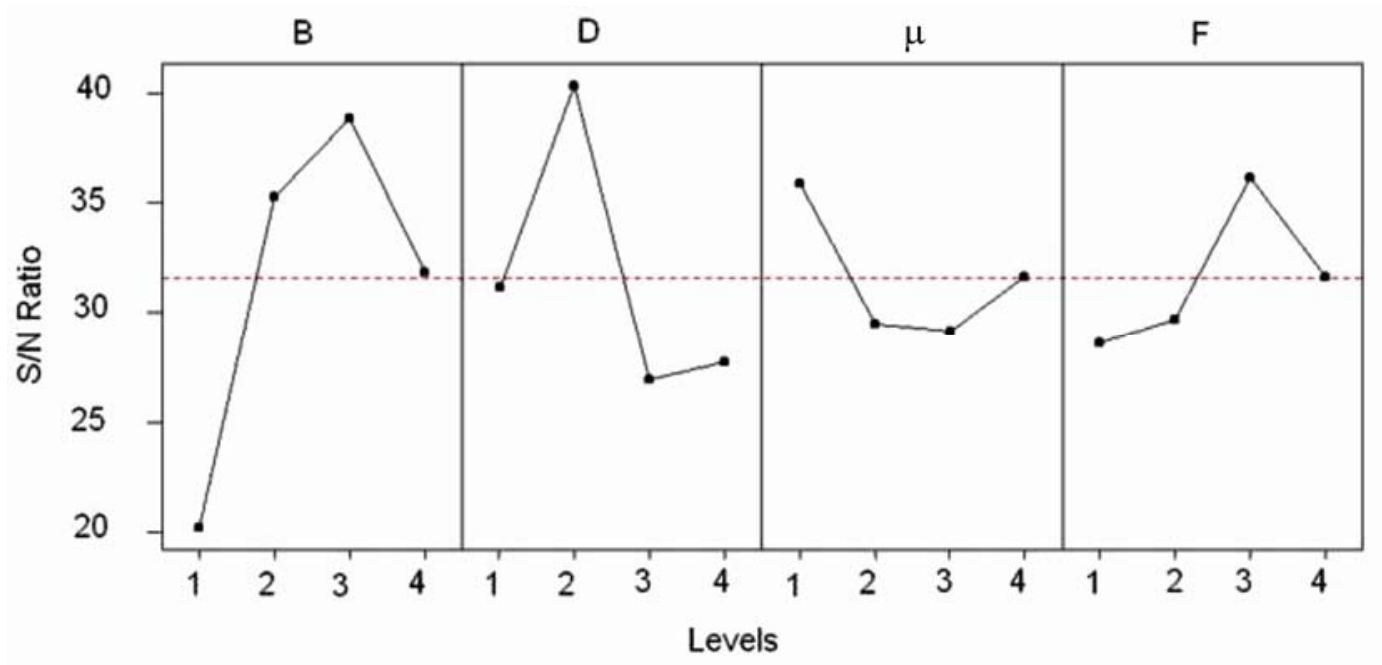

Figure 2. Main effects plot of $S / N$ ratio for measured combustion flame temperature.

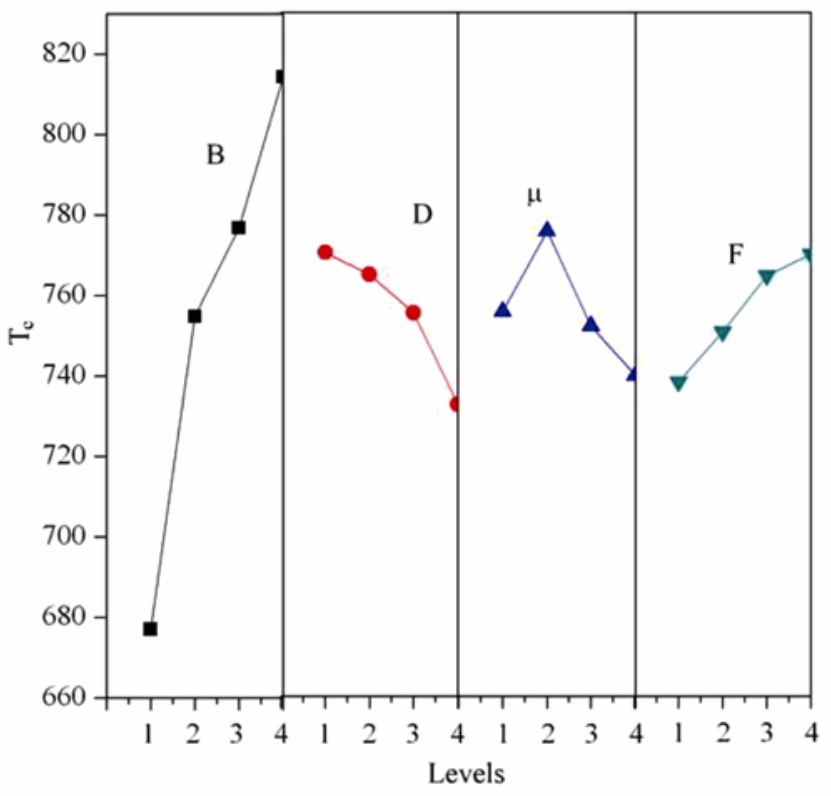

Figure 3. Main effects plot of process parameters on the measured combustion flame temperature.

3.1a Signal-to-noise ratio analysis: Utilizing the experimental data (table 2), and with the help of (2), the $S / N$ ratios for measured combustion flame temperature of each experimental run have been calculated and are displayed in table 2. For the orthogonal experimental design, it is possible to separate out the effect of each process parameter at different levels. For example, the mean $S / N$ ratio for the batch size at levels $1,2,3$ and 4 can be calculated by averaging the $\mathrm{S} / N$ ratios for the experiments $1-4,5-8,9-12$ and $13-16$, respectively. The mean $S / N$ ratio for each level of the other parameters can also be computed in a similar manner. The mean $S / N$ ratio for combustion flame temperature of different selected pro- cess parameters at different levels is shown in table 4. The $S / N$ response plot for combustion flame temperature is shown in figure 2 . The main/direct effect plot for the factors on the response is shown in figure 3 . Irrespective of the quality characteristic chosen for a particular response, a greater $S / N$ ratio corresponds to better quality characteristics. Therefore, the optimal level of the process parameters is the level which ensures greatest $S / N$ ratio. Based on the $S / N$ ratio analysis, the optimal parametric combination for combustion flame temperature becomes $B_{3} D_{2} \mu_{1} F_{3}$ i.e. at levels $3,2,1$ and 3 of factors batch size, diluent, fuel to oxidizer ratio and initial furnace temperature, respectively. It is clear from the $S / N$ ratio analysis (table 4) and effect of process parameters on combustion flame temperature plot (figure 3 ) that the most influencing factor is the batch size followed by diluent, initial furnace temperature and fuel to oxidizer ratio. Batch size and initial furnace temperature have positive effects. With the increase of diluent, the response decreases and for fuel to oxidizer ratio first increases and then follows a decreasing trend.

3.1b Analysis of variance: The purpose of the analysis of variance is to investigate which process parameters have a significant effect on the quality characteristics. In addition, the $F$-test can also be used to determine which process parameters have a significant effect on the measured combustion flame temperature. The result of ANOVA (Roy 2001) is shown in table 5. The percentage contribution of variance, $P$, is calculated as follows:

$$
P=\frac{S S^{\prime}}{S_{\mathrm{T}}}
$$

where $S S^{\prime}$ is pure sum of square and $S_{\mathrm{T}}$ the total sum of square. $F$ ratio of a factor is the ratio of variance of that factor and variance of error. 
Table 4. The mean $S / N$ ratio of process parameters at different levels.

\begin{tabular}{lccccrc}
\hline & \multicolumn{7}{c}{ Mean $S / N$ ratio } \\
\cline { 2 - 7 } Factor & Level 1 & Level 2 & Level 3 & Level 4 & Delta & Rank \\
\hline$B$ & 20.157 & 35.315 & 38.919 & 31.833 & 18.762 & 1 \\
$D$ & 31.214 & 40.380 & 26.893 & 27.736 & 13.487 & 2 \\
$\mu$ & 35.940 & 29.515 & 29.129 & 31.639 & 6.810 & 4 \\
$F$ & 28.609 & 29.714 & 36.216 & 31.684 & 7.607 & 3 \\
\hline
\end{tabular}

Table 5. Result of ANOVA for measured combustion flame temperature.

\begin{tabular}{|c|c|c|c|c|c|c|}
\hline Source & DF & $\begin{array}{c}\text { Sum of } \\
\text { square (SS) }\end{array}$ & $\begin{array}{c}\text { Mean } \\
\text { square (MS) }\end{array}$ & $F$ ratio & $\begin{array}{l}\text { Pure sum } \\
\text { of square }\left(\mathrm{SS}^{\prime}\right)\end{array}$ & $\begin{array}{l}\text { Percent } \\
\text { influence }\end{array}$ \\
\hline$B$ & 3 & 793.406 & 264.469 & $12 \cdot 512$ & 729.993 & $46 \cdot 60$ \\
\hline$D$ & 3 & $457 \cdot 276$ & 152.425 & $7 \cdot 211$ & 393.864 & $25 \cdot 14$ \\
\hline$\mu$ & 3 & $117 \cdot 123$ & $39 \cdot 041$ & $1 \cdot 847$ & $53 \cdot 711$ & $3 \cdot 43$ \\
\hline$F$ & 3 & $135 \cdot 244$ & 45.081 & $2 \cdot 133$ & 71.832 & 4.59 \\
\hline Error & 3 & 63.413 & $21 \cdot 138$ & & & $20 \cdot 24$ \\
\hline Total & 15 & $1566 \cdot 462$ & & & & $100 \cdot 00$ \\
\hline
\end{tabular}

Results of ANOVA (table 5) indicate that the most influential factor is batch size and its percentage contribution is $46 \cdot 6 \%$. This result supports the $S / N$ ratio analysis. The contribution of diluent, $F / O$ ratio and initial furnace temperature are $25.14,3.43$ and 4.59 percent, respectively. The calculated $F$ ratio value has to be compared with $F_{0.05}$ (from standard $F$ tables (Roy 2001) for investigating whether a factor imposes a significant effect on selected response at $\mathbf{9 5 \%}$ confidence levels. A factor is said to have significant effect on a response if the tabulated $F$ value becomes less than the calculated $F$ value. From the $F$ distribution tables (Roy 2001), in relation to the present case, $F_{0.05,3,3}$ is 10.13 at $95 \%$ confidence level. It is evident from table 5 that the effect of batch size becomes significant at both $95 \%$ confidence levels ( $F$ tabulated become less than $F$ calculated). The effect of diluent, fuel to oxidizer ratio and initial furnace temperature are insignificant at $95 \%$ confidence level.

3.1c Verification of results of optimal levels: Once the optimal levels of the design parameters have been selected, the final step of the Taguchi approach is to predict and verify the improvement of the quality characteristic using the optimal parameters combination. The predicted $S / N$ ratio, using the optimal level of the design parameters can be calculated as:

$$
S / N_{\text {predicted }}=S / N_{m}+\sum_{i=0}^{n}\left(S / N_{i}-S / N_{m}\right),
$$

where $S / N_{m}$ is the mean $S / N$ ratio of all the experimental runs, $S / N_{i}$ the mean $S / N$ ratio at the optimal level of $i$ th parameter and $n$ the number of design parameters. Therefore, the predicted $S / N$ ratio can be used to calculate the combustion flame temperature by using following equation:

$$
S / N_{\text {predicted }}=10 \log _{10}\left(\frac{y_{\mathrm{avg}(i)}^{2}}{\sigma_{i}^{2}}\right) .
$$

Table 6 shows comparison of the predicted combustion flame temperature with the experimental result by using the optimal design parameter. The predicted values match well with the experimentally achieved data. The increase of the $S / N$ ratio from the experiment number $10(51.33)$ to the optimal actual data $(56 \cdot 35)$ is about 5.02 dB. This proves the utility of the Taguchi approach in relation to product/process optimization.

\subsection{Mathematical modelling}

The relation between the aqueous solution combustion process parameters, viz. batch size, diluent, fuel to oxidizer ratio and initial furnace temperature, and the process output specified as flame temperature was examined because flame temperature directly controls the phase stability and physical properties of as-formed powder. Three regression models have been developed, such as (i) first degree liner model, (ii) second degree surface model, and (iii) power model. The expressions of these three models are shown in (6), (7), and (8), respectively

$$
T_{c}=a_{0}+a_{1} B+a_{2} D+a_{3} \mu+a_{4} F,
$$

where $a_{i}(i=0 \ldots 4)$ are constants.

$$
\begin{aligned}
T_{c}= & b_{0}+b_{1} B+b_{2} D+b_{3} \mu+b_{4} F+b_{12} B D \\
& +b_{13} B \mu+b_{14} B F+b_{23} D \mu+b_{24} D F \\
& +b_{34} v F+b_{11} B^{2}+b_{22} D^{2}+b_{33} \mu^{2}+b_{44} F^{2},
\end{aligned}
$$

where $b_{i}(i=1 \ldots 4), b_{i j}(i=1 \ldots 4, j=i+0 \ldots 3$ and $j \leq 4)$ are constants. 
Table 6. Result of the confirmation test.

\begin{tabular}{lccc}
\hline & Initial process & \multicolumn{2}{c}{ Optimal process parameters } \\
\cline { 3 - 4 } & parameters & Prediction & Experiment \\
\hline Level & $B_{3} D_{2} \mu_{4} F_{3}$ & $B_{3} D_{2} \mu_{1} F_{3}$ & $B_{3} D_{2} \mu_{1} F_{3}$ \\
Combustion flame temperature $\left(T_{\mathrm{c}}\right)$ & 783 & $782 \cdot 132$ & 782 \\
$S / N$ ratio $(\mathrm{d} B)$ & 51.3265 & 56.7884 & $56 \cdot 3479$ \\
Improvement in $S / N$ ratio $=5.0214$ & & & \\
\hline
\end{tabular}

$$
T_{\mathrm{c}}=c_{0} B^{\mathrm{c}_{1}} D^{\mathrm{c}_{2}} \mu^{\mathrm{c}_{3}} F^{\mathrm{c}_{4}},
$$

where $c_{i}(i=0 \ldots 4)$ are constants.

The regression equations given in (6-8) were solved by using the least square method (Gunaraj and Murugan 1999; Takeshita 2000; Pal et al 2007). The 31 experimental data sets, which were obtained from response surface analysis, were used to develop the models. After evaluation of the coefficients in the regression equations, they are inserted in (6-8), and the final form of the regression model equations are given in (9-11), respectively.

$$
\begin{aligned}
T_{\mathrm{c}}= & 653+7.76 B-1.33 D-12 \cdot 6 \mu+0 \cdot 122 F, \\
T_{\mathrm{c}}= & 746+16.3 B-0.43 D+4.3 \mu-0.515 F \\
& -0.147 B D-1 \cdot 1 B \mu-0.00904 B F+0 \cdot 255 D \mu \\
& +0.00056 D F-0.0297 \mu F+0 \cdot 112 B^{2} \\
& -0.0184 D^{2}+0.11 \mu^{2}+0.000766 F^{2} \\
T_{\mathrm{c}}= & 2.62 B^{0.0753} D^{-0.0338} \mu^{-0.0405} F^{0.0830} .
\end{aligned}
$$

Out of the 15 coefficients (10), the magnitude of coefficients of the second order interaction term is negligible compared to those of the process variables viz. batch size $(B)$, diluents $(D)$, fuel to oxidizer ratio $(\mu)$ and initial furnace temperature $(F)$. Thus the major contribution to the flame temperature of combustion reaction comes from these variables. Secondly the sign of coefficient of batch size and fuel to oxidizer ratio is positive whereas sign of coefficient of initial furnace temperature and diluents is negative. It means, with the increase of $B$ and $\mu$, the flame temperature becomes more and with the increase of $F$ and $D$, the flame temperature becomes less and that is what is expected. Flame temperature of the process directly controls the phase formation and physical properties of the as-formed HAp powder. According to (10), it is clear that higher values of $B$ and $F$ may increase the crystallite size whereas higher values of $\mu$ and $D$ may reduce the crystallite size of as-formed HAp powder. The experimental results were verified in terms of phase formation and crystallite size of HAp powder with flame temperature and the result was quite expected. 3.2a Adequacy of developed mathematical models: The adequacy of the developed models was tested using the ANOVA technique. ANOVA has been performed in the statistical software package MINITAB (MINITAB ${ }^{\mathrm{TM}}$, USA, 2000). It uses the $P$-value, termed as probability of significance. $P$-value is calculated based on calculated $F$ value. $P$-value thus obtained is then compared with the Alpha-level. The presumed Alpha-level depends on the confidence level (say 95\%) chosen. If the $P$-value appears $<0.05$, then it can be concluded that the model is adequate within the confidence limit. ANOVA test results are presented in tables 7-9 for linear, surface and power models, respectively. From the tables, it can be understood that the developed mathematical model is found to be adequate to predict the flame temperature of aqueous solution combustion process at $95 \%$ confidence level. Coefficient of correlation ' $R$ ' ' is used to find how close the predicted and experimental values lie. The values of $R^{2}$ for the above developed models are shown in their corresponding ANOVA table. The correlation graph (scatter diagram of experimental vs model predicted values) of flame temperature for linear, surface and power models are shown in figures 4-6, respectively. Among these three models, $R^{2}$ value of second degree surface equation is near to unity, which indicates high correlation between the experimental values and predicted values, and this is further supported by correlation graph. Therefore, this model is considered best for representing input-output relationship of aqueous solution combustion process.

\subsection{Product characteristics}

3.3a Phase analysis of as-synthesized powder: Typical X-ray diffraction patterns of combustion synthesized powders obtained from different experimental conditions are shown in figure 7. The as-synthesized powders were found to be well crystalline in most of the cases and identified as pure hydroxyapatite phase (by JCPDS file 090432). These experimental results indicate that the well crystalline HAp may be directly formed if the combustion flame temperature exceeds above $750^{\circ} \mathrm{C}$.

Below that temperature as synthesized powders are amorphous in nature which is shown in figure 7(e). The crystallite size of as-synthesized powder was measured from the X-ray peak broadening of the (211) diffraction 
Table 7. Analysis of variance tests for linear model.

\begin{tabular}{lrccccc}
\hline Source & DF & $\begin{array}{c}\text { Sum of } \\
\text { square (SS) }\end{array}$ & $\begin{array}{c}\text { Mean } \\
\text { square }(\mathrm{MS})\end{array}$ & $\begin{array}{c}\text { Pure sum of } \\
\text { square }\left(\mathrm{SS}^{\prime}\right)\end{array}$ & $F$ ratio & $P$ \\
\hline Eq. 9 & 4 & $16349 \cdot 7$ & 4087.42 & 16349.7 & 48.61 & $<0 \cdot 001$ \\
Linear & 4 & 16349.7 & 4087.42 & 16349.7 & 48.61 & $<0.001$ \\
Residual error & 26 & $2186 \cdot 0$ & 84.08 & 2186.0 & & \\
Lack-of-fit & 20 & $2077 \cdot 2$ & 103.86 & $2077 \cdot 2$ & 5.72 & 0.091 \\
Pure error & 6 & 108.9 & $18 \cdot 14$ & 108.9 & & \\
Total & 30 & 18535.7 & & & & \\
\hline
\end{tabular}

$R^{2}=88.2 \%, R^{2}$ (adj) $=86.4 \%$ and model is adequate

Table 8. Analysis of variance tests for surface model.

\begin{tabular}{|c|c|c|c|c|c|c|}
\hline Source & $\mathrm{DF}$ & $\begin{array}{c}\text { Sum of } \\
\text { square (SS) }\end{array}$ & $\begin{array}{c}\text { Mean } \\
\text { square (MS) }\end{array}$ & $\begin{array}{l}\text { Pure sum } \\
\text { of square }\left(\mathrm{SS}^{\prime}\right)\end{array}$ & $F$ ratio & $P$ \\
\hline Eq. 10 & 14 & $17519 \cdot 3$ & $1251 \cdot 38$ & $17519 \cdot 3$ & $19 \cdot 7$ & $<0.001$ \\
\hline Linear & 4 & $16349 \cdot 7$ & 34.48 & 137.9 & $0 \cdot 54$ & 0.707 \\
\hline Square & 4 & $369 \cdot 2$ & $92 \cdot 31$ & $369 \cdot 2$ & 1.45 & $0 \cdot 262$ \\
\hline Interaction & 6 & $800 \cdot 4$ & $133 \cdot 4$ & $800 \cdot 4$ & $2 \cdot 10$ & $0 \cdot 110$ \\
\hline Residual error & 16 & $1016 \cdot 4$ & 63.52 & $1016 \cdot 4$ & & \\
\hline Lack-of-fit & 10 & 907.5 & $90 \cdot 75$ & 907.5 & 5.00 & 0.031 \\
\hline Pure error & 6 & $108 \cdot 9$ & $18 \cdot 14$ & $108 \cdot 9$ & & \\
\hline Total & 30 & 18535.7 & & & & \\
\hline
\end{tabular}

$R^{2}=94.5 \%, R^{2}$ (adj) $=89.7 \%$ and model is adequate

Table 9. Analysis of variance tests for power model.

\begin{tabular}{lrccccc}
\hline Source & DF & $\begin{array}{c}\text { Sum of } \\
\text { square (SS) }\end{array}$ & $\begin{array}{c}\text { Mean } \\
\text { square (MS) }\end{array}$ & $\begin{array}{c}\text { Pure sum of } \\
\text { square }\left(\mathrm{SS}^{\prime}\right)\end{array}$ & $F$ ratio & $P$ \\
\hline Eq. 11 & 4 & 0.00598 & 0.0015 & 0.00598 & 49.67 & $<0.001$ \\
Linear & 4 & 0.00598 & 0.0015 & 0.00598 & 49.67 & $<0.001$ \\
Residual error & 26 & 0.00078 & 0.00003 & 0.00078 & & \\
Lack-of-fit & 20 & 0.00074 & 0.00004 & 0.00074 & 5.51 & 0.021 \\
Pure error & 6 & 0.00004 & 0.0 & 0.00004 & & \\
Total & 30 & 0.00676 & & & & \\
\hline
\end{tabular}

$R^{2}=88.4 \%, R^{2}($ adj $)=86.6 \%$ and model is adequate

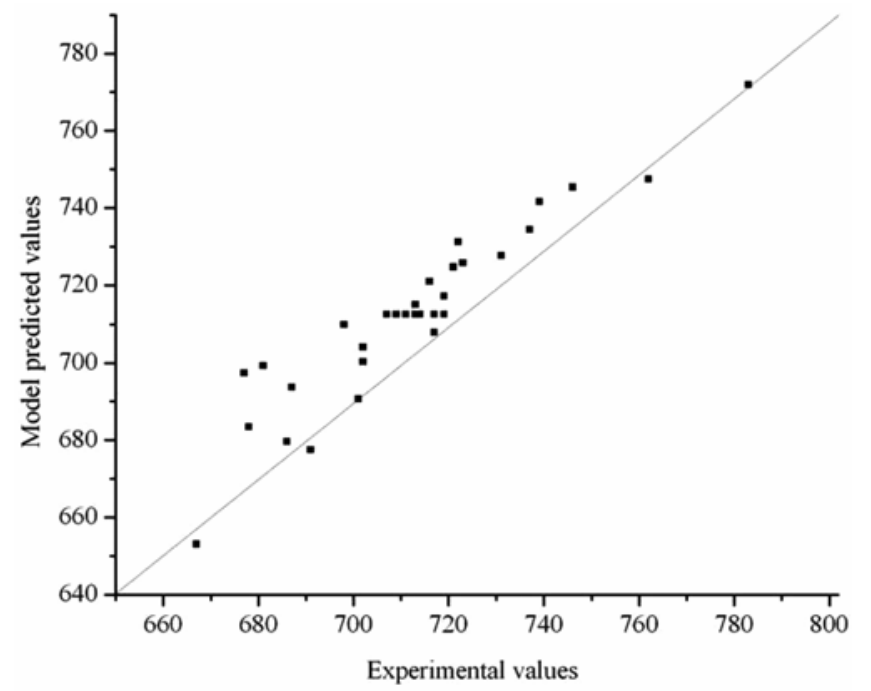

Figure 4. Correlation graph of measured combustion flame temperature for linear model.

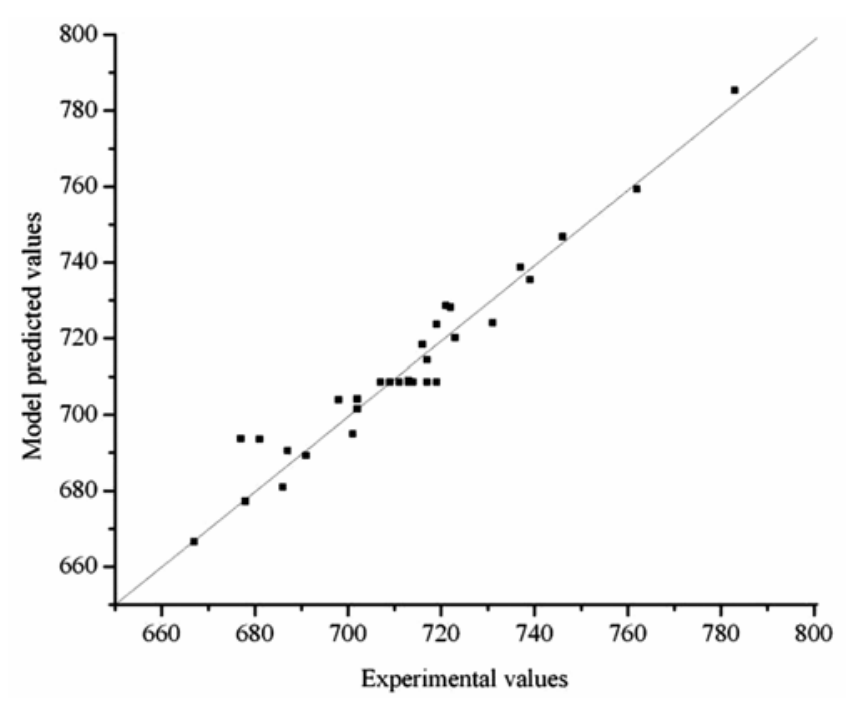

Figure 5. Correlation graph of measured combustion flame temperature for surface model. 


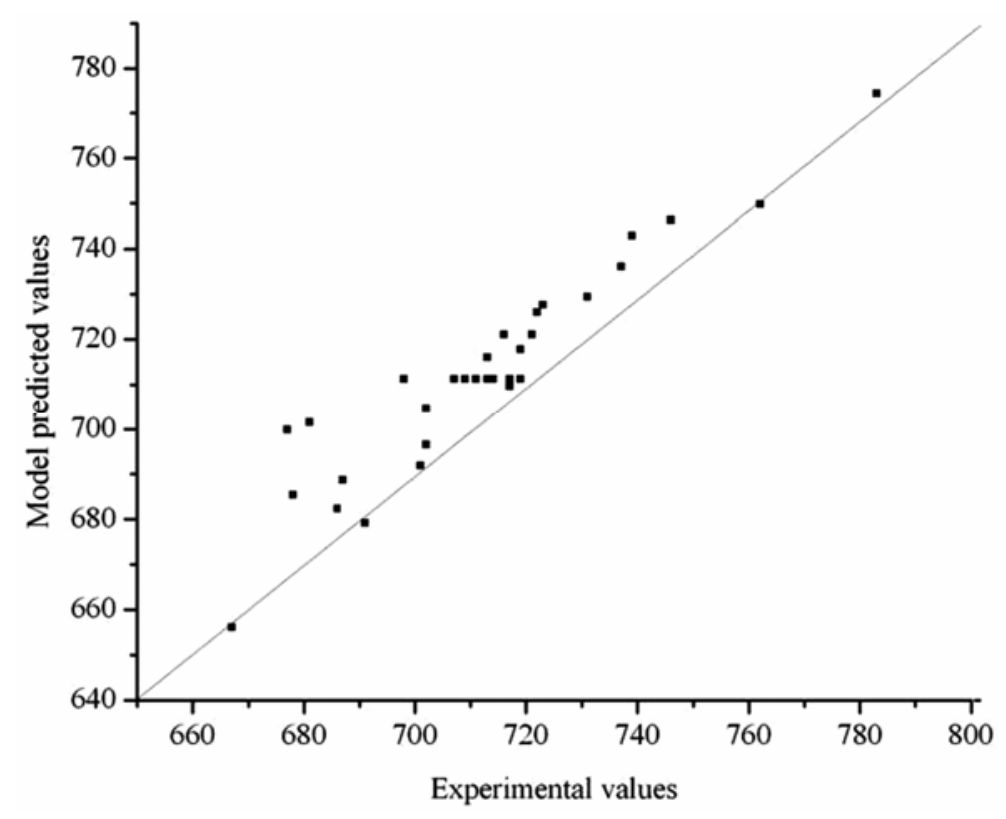

Figure 6. Correlation graph of measured combustion flame temperature for power model.

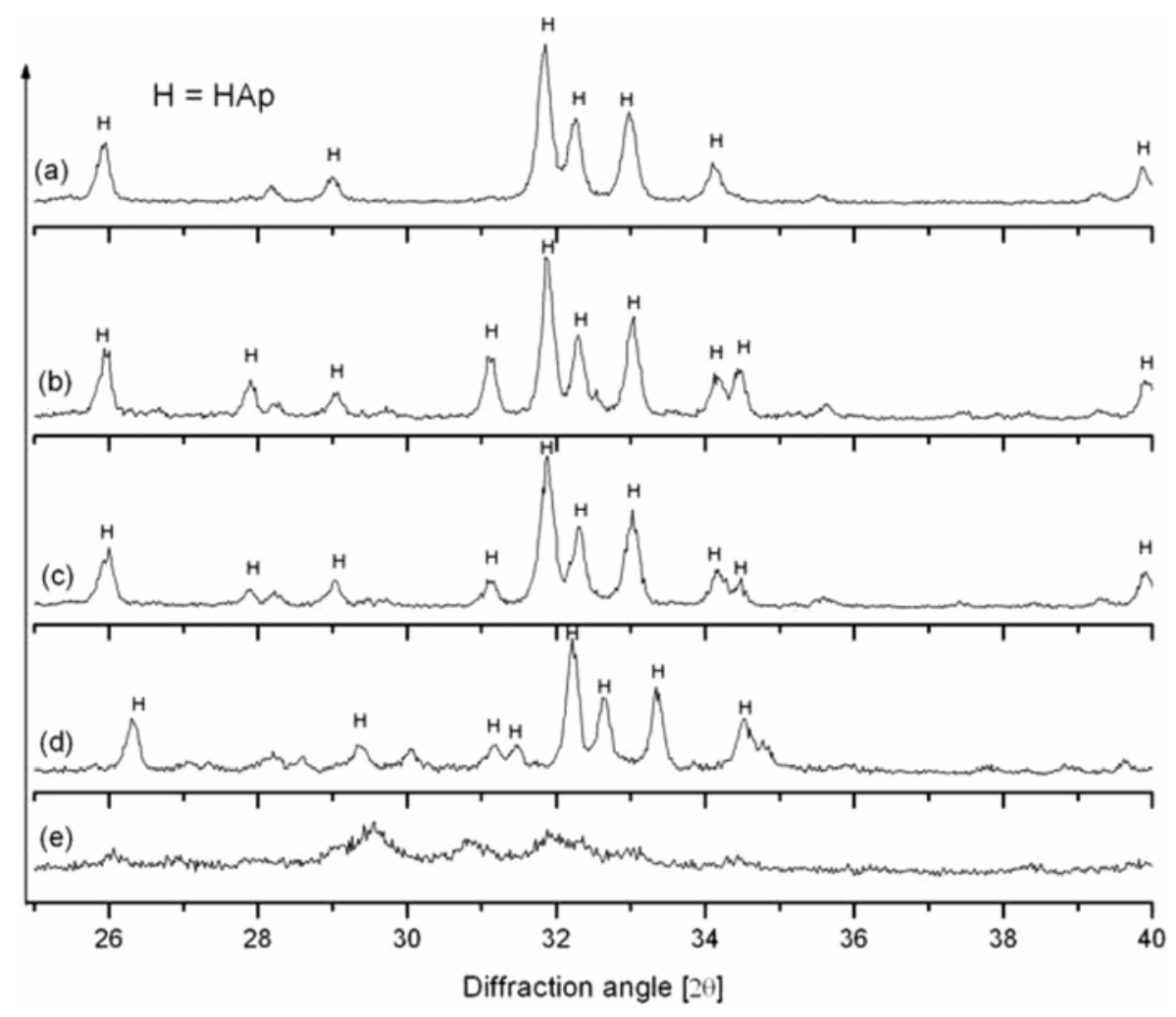

Figure 7. Represents the X-ray diffraction patterns of as-synthesized powders.

peak of HAp by using standard Scherrer's formula. Figure 8 shows the flame temperature versus crystallite size of as-synthesized powders. It was found that crystallite size of as-formed powder significantly increases with increase in flame temperature of the combustion reaction.
Precursors having combustion flame temperatures (figures d-a) $783,797,807$ and $852^{\circ} \mathrm{C}$ produced HAp powder with the crystallite size $47.8,50 \cdot 0,53.0$ and $64.5 \mathrm{~nm}$, respectively. It was evident that the crystallite size of the as-formed HAp powder depends on the flame temperature 


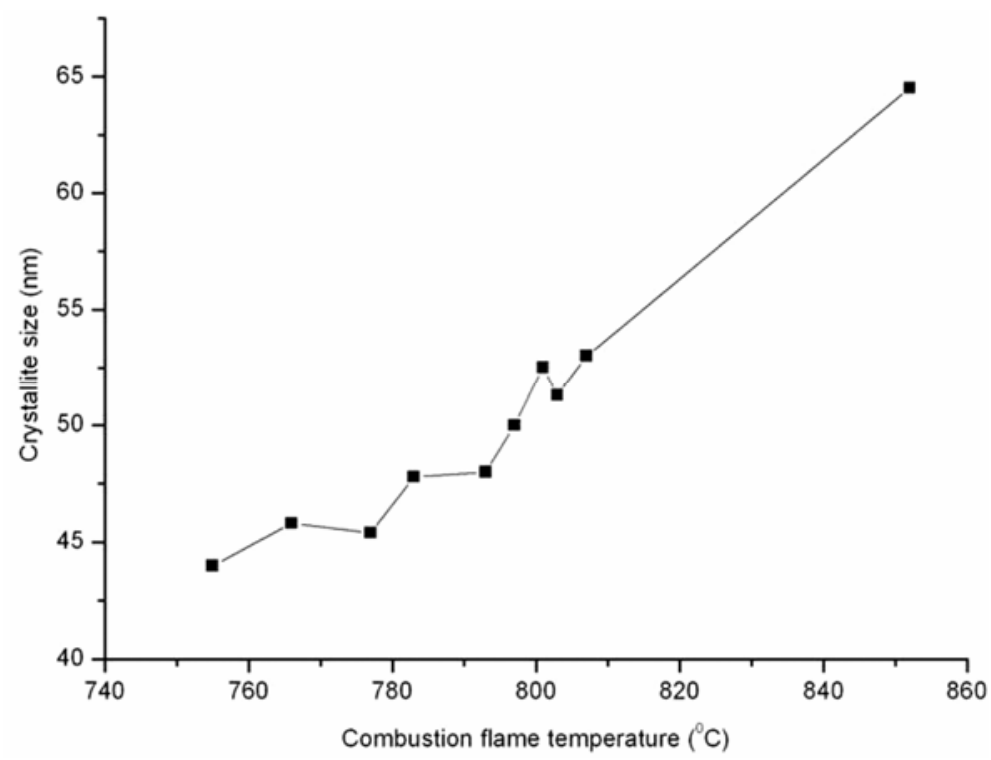

Figure 8. Shows influence of combustion flame temperature on the crystallite size of the as-formed HAp powder.

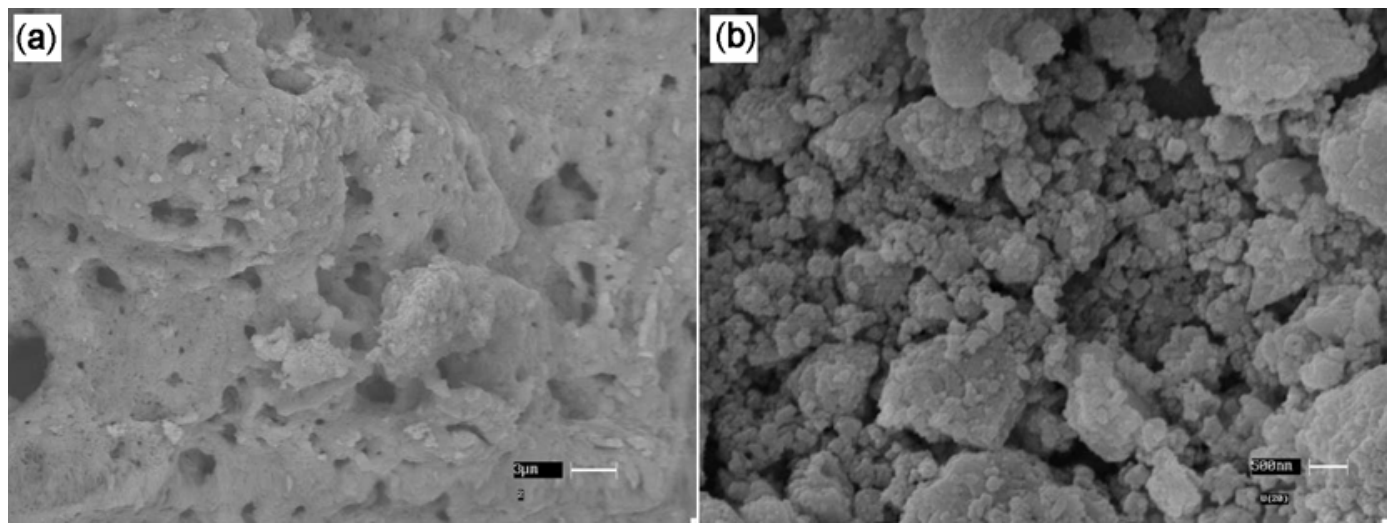

Figure 9. (a)-(b) Showing SEM micrographs of experiment run 13.

as well as nature of the combustion reaction. Higher flame temperature leads to sintering of the particles locally that increased the crystallite size of HAp powder.

Powder morphology of as-formed powders was characterized by SEM technique. It exhibited foamy agglomerated particles with homogeneous distribution and presence of random distribution of voids in their structure. This highly porous, foam-like structure was formed due to the inherent nature of the chemical reaction associated with the evolution of large volume of gases, and short reaction period followed by fast quenching which prevented further agglomeration/sintering of particles. Figure $9(\mathrm{a}, \mathrm{b})$ shows the SEM micrographs of as-formed powder. Micrographs show that at first spherical nanoparticles formed under experimental condition undergoes extensive agglomeration. Some isolated spherical particles detected exhibit a size of $<50 \mathrm{~nm}$.

\section{Conclusions}

In the present study, a detailed methodology of the Taguchi optimization technique has been reported and applied for evaluating optimal parametric combinations to achieve a target flame temperature in solution combustion synthesis of calcium hydroxyapatite powder. The optimum combination of experimental variables for phase pure nanocrystalline HAp powder is $B_{3} D_{2} \mu_{1} F_{3}$ i.e. at levels $3,2,1$ and $3, T_{\mathrm{c}}=783^{\circ} \mathrm{C}$, crystallite size $=47.8 \mathrm{~nm}$. The $S / N$ ratio and ANOVA test both have shown that the batch size is a very important controlling factor followed by diluent, initial furnace temperature and fuel to oxidizer ratio. In addition to this, RSM technique has also been used to develop regression model to correlate flame temperature with process parameters. The developed threeregression model was found to be adequate to predict the 
flame temperature of aqueous solution combustion process at $95 \%$ confidence level. $R^{2}$ value of second degree surface model become more than the other two developed models; therefore, this model is considered the best for representing input-output relationship of aqueous solution combustion process. Additionally it has been shown that with the decrease of batch size and initial furnace temperature and increase of fuel to oxidizer ratio and diluents, the crystallite size of as-formed HAp decreases.

\section{Acknowledgements}

The authors would like to acknowledge Mrs Archana Pal, Asansol, West Bengal, India, for her valuable suggestions to present the statistical tools properly. The authors wish to thank Dr H S Maiti, Director, Central Glass and Ceramic Research Institute, Kolkata, for his keen interest and constant encouragement.

\section{References}

Balmer M L, Lange F F, Jayaram V C and Levi G 1995 J. Am. Ceram. Soc. 781489

Fanovich M A, Castro M S and Porto Lopez J M 2001 Mater. Res. Bull. 36487

Feng W, Li Mu-sen L, Yu-peng Q and Yong-xin A 2005 Mater. Letts $\mathbf{5 9} 916$

Fumo D A, Morelli M R and Segadaes A M 1996 Mater. Res. Bull. 311243

Ghosh S K, Datta S and Roy S K 2004 Trans. Indian Ceramic Soc. 6327

Ghosh S K, Nandi S K, Kundu B, Datta S, De D K, Roy S K and Basu D 2008 J. Biomed. Mater. Res.: Part B-Appl. Biomater. B86 217

Gunaraj V and Murugan N 1999 J. Mater. Process. Technol. 88 266

Hench L L 1991 J. Am. Ceram. Soc. 741487

Hench L L 1998 J. Am. Ceram. Soc. 811705

Hing K A, Best S M and Bonfield W 1999 Mater. Sci: Mater. Med. 10135

Hong C S, Ravindranathan P, Agrawal D K and Roy R 1994 J. Mater. Res. 92398

Jain S R, Adiga K C and Vernekar V R P 1981 Combust. Flame 4071

Kanazawa T 1989 Inorganic phosphate materials, materials science monographs (Tokyo: Elsevier Science Publishers; Amsterdam: Kodansha Ltd.) Vol. 52, pp. 55-77
Kingsley J J and Patil K C 1988 Mater. Lett. 6427

Liu H S, Chin T S, Lai L S, Chiu S Y, Chung K H, Chang C S and Liu M T 1997 Ceram. Int. 2325

Liu D M, Troczynski T and Tseng W J 2001 Biomaterial 22 1721

Liu D M, Yang Q, Troczynski T and Tseng W J 2002 Biomaterials 231679

Manoharan S S and Patil K C 1992 J. Am. Ceram. Soc. 751012

Minitab Inc., User manual of MINITAB ${ }^{\mathrm{TM}}$ Statistical Software, Release 13.31, State College, PA 16801 USA, 2000

Mukasyan A S, Costello C, Sherlock K, Lafarga P D and Varma A 2001 Sep. Purif. Technol. 25117

Myers R H and Montgomery D C 1995 Response surface methodology: process and product optimization using designed experiments (New York: John Wiley \& Sons Inc.)

Nandi S K, Ghosh S K, Kundu B, De D K and Basu D 2008a Small Rumi. Res. 75144

Nandi S K, Kundu B, Ghosh S K, De D K and Basu D 2008b J. Vet. Sci. 9183

Nandi S K, Kundu B, Ghosh S K, Mandal T K, Datta S and Basu D 2009 Ceram. Int. 351367

Pal S, Pal S K and Samantaray A K 2008 J. Mater. Proc. Technol. 202464

Purohit R D, Sharma B P, Pillai K T and Tyagi A K 2001a Mater. Res. Bull. 362711

Purohit R D, Saha S and Tyagi A K 2001b J. Nucl. Mater. 2887

Raemdonck W, Ducheyne V P and Meester P D 1984 Metal and ceramic biomaterials (eds) P Ducheyne and W Hasting (Boca Raton, Florida: CRC Press) Vol. 2, pp 149-154

Roy R K 2001 Design of experiments using the Taguchi approach (New York: John Wiley \& Sons Inc.)

Schafer J, Sigmund W, Roy S and Aldinger F 1997 J. Mater. Res. 122518

Segadges A M, Morelli M R and Kiminami R G A 1998 J. Eur. Ceram. Soc. 18771

Suchanek W L, Shuk P, Byrappa K, Riman R E, TenHuisen K S and Janas V F 2002 Biomaterials 23699

Sung Y, Lee M J C and Yang J W 2004 J. Cryst. Growth 262 467

Tas A C 2000 J. Eur. Ceram. Soc. 202389

Takeshita K 2000 Weld. J. 79 261s

Varma H K, Kalkura S N and Sivakumar R 1998 Ceram. Int. 24 467

Venkatachari K R, Huang D, Ostrander S P and Schulze W A 1995 J. Mater. Res. 10748

Wang P E and Chaki T K 1993 J. Mater. Sci: Mater. Med. 450

Willman G 1996 Br. Ceram. Trans. 95212

Zahid A 1998 Calcium phosphates in biological and industrial systems (Boston, USA: Kluwer Academic Publisher) pp 21-24 